\title{
SDN Controller Deployment for QoS Guarantees in Tactical Ad Hoc Networks
}

\author{
Xin Yan ${ }^{D},{ }^{1}$ Xiaodong $\mathrm{Hu}^{2}$ and Wen Liu ${ }^{1}$ \\ ${ }^{1}$ School of Computer Science \& Technology, Wuhan University of Technology, Wuhan 430070, China \\ ${ }^{2}$ School of Mathematics \& Computer Science, Wuhan Textile University, Wuhan 430200, China \\ Correspondence should be addressed to Xin Yan; yanxin@whut.edu.cn
}

Received 6 March 2021; Accepted 4 May 2021; Published 15 May 2021

Academic Editor: Fangqing Wen

Copyright ( 2021 Xin Yan et al. This is an open access article distributed under the Creative Commons Attribution License, which permits unrestricted use, distribution, and reproduction in any medium, provided the original work is properly cited.

\begin{abstract}
Tactical ad hoc networks (TANET) accomplish the corresponding tasks via a hopeful device-to-device connection mechanism for data transmission and resource management without a centralized foundation. Software-defined networking (SDN) provides an evolution from the previous networks by decoupling the network control from data forwarding and providing a novel paradigm for network handling. Nevertheless, a SDN-based strategy in TANET leads to various novel problems since the primary construction cannot be employed in mobile ad hoc networks anymore. In this paper, a new SDN-based structure is constructed for TANET. Then, both delay and energy consumption (EC) are utilized to model the controller deployment and data-plane assignment problems, by which quality-of-service (QoS) guarantees are realized. Afterwards, mixed-integer programming (MIP) is adopted to solve the mentioned model. Finally, according to the experimental outcomes, it can be observed that the presented approach ensures the data-plane delay and optimizes the EC.
\end{abstract}

\section{Introduction}

With the increasing growth of the technology of wireless communications, data traffic transmitted through wireless networks is continuously increasing. Wireless mobile communication networks commonly contain wireless local area networks, cellular networks, mobile ad hoc networks, wireless mesh networks, etc. Local traffic may be caused when the source and the destination are close. In popular network situations, local traffic transmission and redistribution can be realized from the cellular network to the main structure. As a more suitable strategy, a distributed organization for devices can be realized using device-to-device technologies. Lack of centralized foundation leads to independent operation, making routing decisions, and dynamic adaptation of each node to topology variations in a mobile network. The mentioned features lead to significant achievements for mobile ad hoc networks in a tactical network scenario, in other words, tactical ad hoc networks (TANET) [1].

As a hopeful novel pattern, software-defined networking (SDN) makes a remarkable change in the construction and operation of communication networks. Due to its universal view on the whole network, the centralized SDN controller leads to a remarkable improvement in its service quality and management of resources. SDN adopts task-specific benchmark and necessary to know restrictions within the tactical networks to make globally optimal routing decisions and provide data transmission. The SDN has been employed in ad hoc networks to improve efficiency and flexibility [2]. In comparison to the traditional distributed routing procedures (like optimized link state routing, OLSR), the SDNenabled structure leads to lower response delay, superior throughput, and energy consumption (EC).

SDN provides an improvement in management intelligence and flexible control in TANET. Nevertheless, due to the distribution of resources and management in mobile ad hoc networks, employing a centralized SDN pattern in TANET (i.e., software-defined tactical ad hoc networks, SD-TANET) might be complicated. For the SDN unit's proper operation, specific communications should be established among controllers and data-plane services using network state data (e.g., topology discovery) and flow tables. 
The mentioned communications can be influenced in TANET due to the low transfer rates and unreliable link connection. Due to the intrinsic limitations for devices in SDTANET, the controllers should be distributed within the network to achieve superior performance and reliability.

A redundancy is incorporated into the control plane to overcome the mentioned deficiencies and guarantee the connection reliability among data-plane nodes and controllers. In a large-scale SD-TANET, multiple controller deployment can be considered an appropriate approach. Due to the critical role of the SDN controller within the network and the movement of the energy-intensive functions from nodes to the controller, the SDN controller's optimum deployment has a considerable impact on decreasing the response delay and EC. An essential issue here is to decide where to locate multiple controllers in the network. This decision is able to significantly affect the efficiency of the SDN-based TANET.

The other sections of the present article are classified as given below: Section 2 is allocated to the relevant studies, which employ SDN in mobile ad hoc networks and the issues of wireless controller deployment. In Section 3, the presented design and SDN-based TANET are introduced, which are adopted to describe the deployment issue of controllers and the data-plane assignment to the controllers in Section 4. The solution of the mentioned model is given in Section 5, and the presented approach is evaluated through a practical dataset. The obtained conclusions are drawn in Section 6.

\section{Relevant Studies}

Current controller software, including ONOS [3] and the software switch Open vSwitch [4], could be implemented on various instruments, even certain weightless network ones. This makes it feasible to employ SDN in mobile ad hoc networks. Besides, various controller deployment approaches can be realized using common software execution.

In [5], the authors introduced a feasible implementation of an SDN-based MANET (namely, mobile ad hoc networks) and characterized the developed software components. The authors in [6] constructed a framework and a related prototype for SDN-based quality management, which provides high flexibility via novel flow management rules at preparation time and can appropriately handle node join/leave events. We see in [1] a structure constructed for SDN-based mobile ad hoc networks in the tactical area and illustrated the difficulties caused via the ad hoc and coalition network ambience. In [7], the technology of SDN has been employed for the ad hoc sensor networks of flying, and a clustering structure with a clustered controller of SDN is constructed to realize hierarchical management and integrated dispatch. In [8], a flexible procedure has been presented, which could dynamically select to route under the controller's commands or make routing choices through a distributed approach. The mentioned studies demonstrate the possi- bility of employing SDN in ad hoc networks and its functionality aspects in tactical field networks.

Controller deployment problem (CPP) can spread out a fair quantity of controllers at optimum positions. Several studies have been performed about wired networks based on various targets and limitations. Nodal mobility and channel uncertainty are two essential features of the wireless network, imposing unique CPP in software-defined wireless networks (SDWN) [9]. The CPP issue has been considered in SDWN in various studies.

The authors in [10] presented a TDMA-based controller, in which wireless control plane and southbound interfaces have been considered to diminish the quantity of controllers in a specified controller reaction time. We see in [11] a novel assessment named transparency and optimized multiple goals taking channel conflict and packet loss in wireless networks. In [12], the authors verified the influence of delayed state data and constructed a deployment structure of dynamic controller, where delayed queue length data has been utilized to relocate the controller.

The data mentioned above give us the possibilities of not only employing SDN in TANET but also implementing CPP with wireless links. Although the CPP with delay or packet loss guarantee in a wireless scenario has been studied, the issue with multiple QoS constraints has not been involved yet. In particular, EC constraint is a crucial QoS guarantee for both controllers and data nodes in wireless mobile networks. Thus, in addition to channel conflict, both delay and EC are considered in this paper, which is our major contribution as well.

\section{The SD-TANET Structure}

The constructed structure of SDN-based TANET and their operation is illustrated in the current section. An appropriate MAC layer should be selected in the data plane for the TANET organization. Unlicensed bands without centralized coordination determine the mentioned selection rule for the MAC protocol. Based on [5], IEEE 802.11 P2P mode independent basic service set (IBSS) is the ad hoc mode standard of IEEE 802.11, providing complete control on the upper layers, which is necessary for the utilized method.

Although the SDN is founded on the decoupling of the data and control planes, it is more reasonable to employ a hybrid structure in the SD-TANET for the control plane. In a hybrid structure, data transmission via data-plane nodes could be realized using the flow tables transmitted via controllers. If the target node is undetectable or the flow tables are not updated by the controller, the old flow tables could be neglected via the data-plane nodes, and messages can be transmitted via distributed routing rules. This design kind causes data-plane nodes to create distributed routing choices to improve the control plane's redundancy.

Further necessity in this architecture is to employ two distinct frequency bands to split network control and data sending, enhancing the scalability and performance. The 


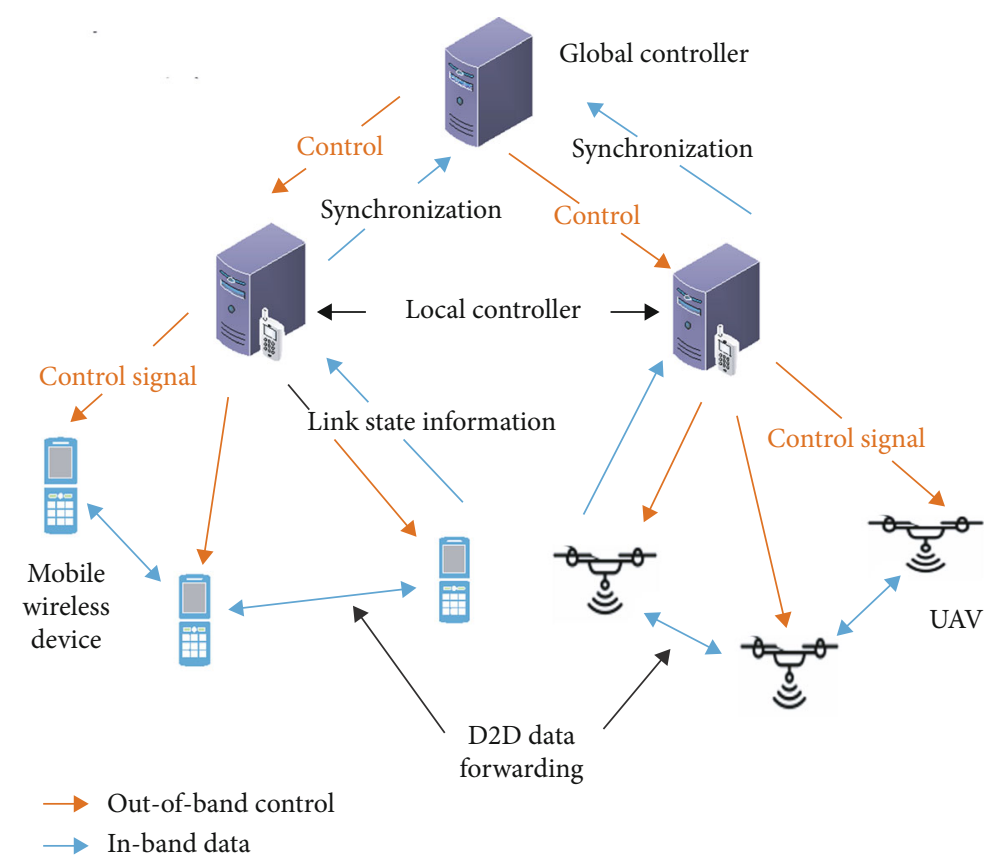

FIGURE 1: The SD-TANET structure, where the mobile wireless instruments and unmanned aerial vehicles (UAV) with SDN capability create the data plane.

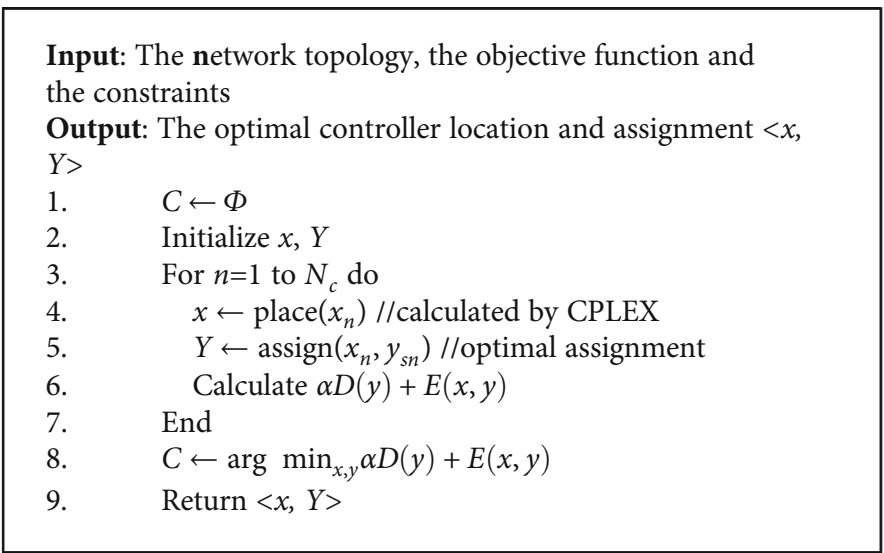

Algorithm 1: The controller deployment and data-plane assignment.

"in-band" phrase is employed for point-to-point data transfer among distributed devices, while the "out-of-band" phrase is utilized for point-to-multipoint control signaling among the controller and distributed devices [13, 14]. Figure 1 describes the details of the hybrid structure, which consists of a global controller and some local controllers, forming the control plane.

An instrument with adequate processing of data abilities is able to operate as the local controller, deployed on a portable wireless foundation or SDN-based mobile instruments. It combines link-state data with local topology from all corresponding distributed instruments. The global controller provides a general perspective of the network and preprocesses link-state data with a centralized strategy. It can be spread out in an edge computing center like the center of commanding in the networks of tactical field. The local controller operates as a link connecting the global controller to distributed devices. Optimal routing tables obtained via the global controller are sent via the local controller. The local controller can perform the overall control under a global controller's failure or real-time response requirements. The routing of devices can be realized using their processed data.

\section{System Model and Strategy}

The control plane consists of the controllers' deployment and the data-plane nodes' assignment. The network, especially the response delay and EC for transmission and synchronization, can be effectively influenced by the control-plane structure. The following challenges can be solved using the presented methodology: (1) the number of controllers; (2) 


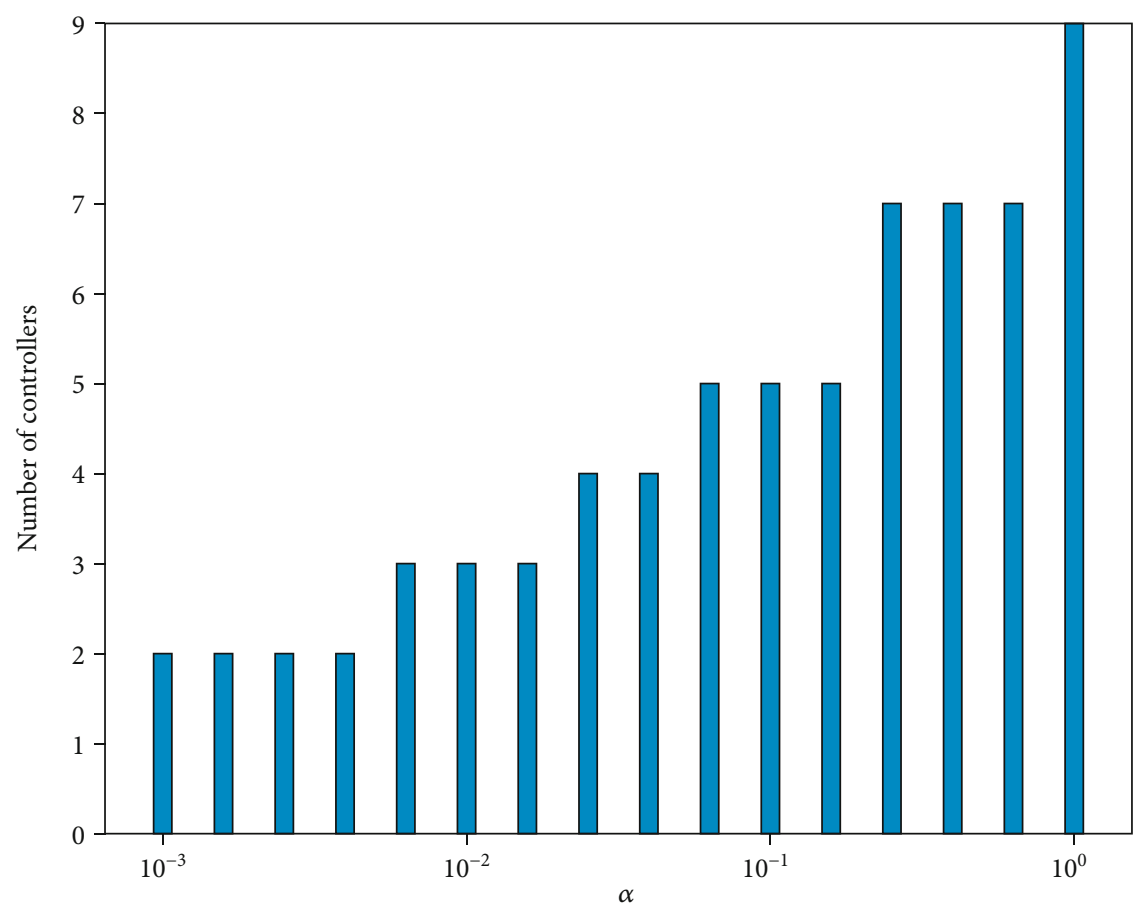

FIgURE 2: Quantity of controllers for various weight values $\alpha$.

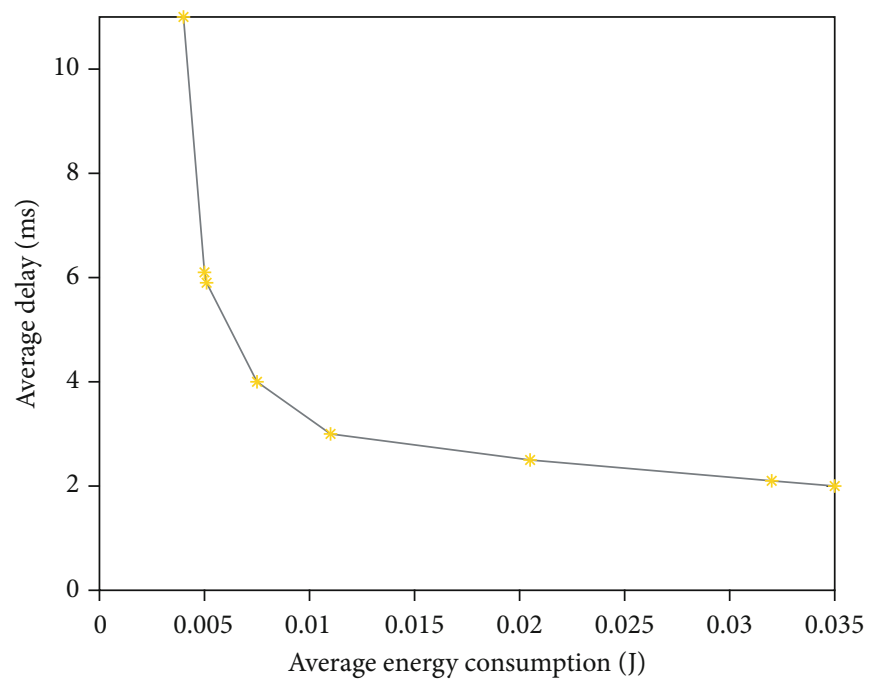

Figure 3: Trade-off among EC and delay.

the controller position; and (3) the assignment of data-plane nodes to the controller.

A network is described with $G(N, E)$. Suppose that the data-plane nodes and controllers are indicted with the set $N$. The wireless connection among data-plane nodes and controller is described by set $E$.

As shown in Figure 1, the smartphones and unmanned aerial vehicles (UAV) could be described via data-plane nodes if they are SDN-consistent and support the MAC layer explained in the prior section. Virtual SDN switches can be simulated with the network nodes. The mentioned mobile ad hoc nodes and the controller set are denoted by
$S=\left\{s_{1}, s_{2}, \cdots, s_{k}\right\}$, and $C=\left\{c_{1}, c_{2}, \cdots, c_{t}\right\}$, respectively. Consider that all TANET nodes can operate as a controller. A series of nodes with sufficient resources that are able to operate as a controller is denoted by $N_{c} \subseteq N$.

Now, $x_{c} \in\{0,1\}$ is defined as binary decision variables, as the following (1), indicating whether a local controller is positioned at node $c \in N_{c}$. The mentioned variables can be employed to develop a controller deployment approach.

$$
x=\left(x_{n} \in\{0,1\}: n \in N\right) .
$$




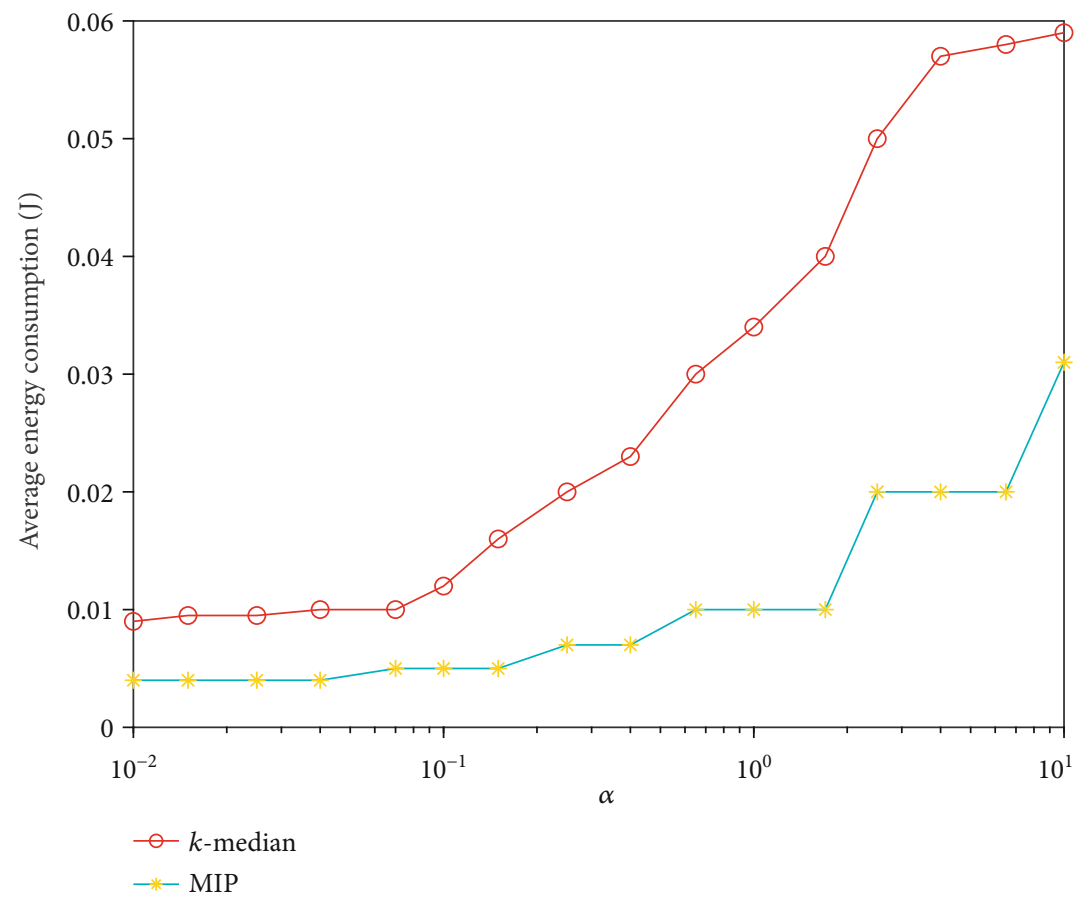

FIgURE 4: Comparison with the $k$-median approach.

Since a controller should be positioned at a node with sufficient resources, the following condition should be fulfilled:

$$
x_{n}=0, \quad \forall n \notin N_{c} .
$$

The presented approach should determine the assignment of data-plane nodes to controllers. This implies that an appropriate controller must be selected to manage it. In a similar manner, decision variables $y_{s c} \in\{0,1\}$ indicate whether a node $s \in S$ corresponds to the controller at $c \in$ $N_{c}$ and $y_{s c}=1$ or not $y_{s c}=0$. The assignment rule is described as

$$
y=\left(y_{s c} \in\{0,1\}: s \in S, c \in N_{c}\right) .
$$

Since any data-plane node must be devoted to an individual controller at a determined time, the following constraint should be satisfied:

$$
\sum_{c \in N_{c}} y_{s c}=1, \quad \forall s \in S
$$

As another constraint, if node $s$ corresponds to controller $c$, controller $c$ should be necessarily positioned there. Thus, the following constraint should be fulfilled:

$$
y_{s c} \leq x_{c}, \quad \forall s, c \in N
$$

The presented controller deployment and assignment strategy's primary purpose is to decrease the reaction delay and the communication overhead for state data collection and synchronization. Then, the model of delay and overhead can be studied.

It can be seen from Figure 1 that control and data planes send control signals and status data through wireless links. Any wireless link is related to probability $p_{s c}$, which defines the probability that a message can be successfully transmitted on the mentioned link. $p_{s c}$ is dependent on the model of wireless channel that considers a combined path loss and shadowing model, presented through [15].

$$
p_{s c}=Q\left(\frac{P_{\min }-\left(P_{\mathrm{t}}+10 \lg k-10 \gamma \lg \left(d / d_{0}\right)\right)}{\sigma_{\psi_{d B}}}\right) .
$$

$Q$ describes the probability that a Gaussian random variable $x$ with the zero mean and the unit variance can be higher than $z$ :

$$
Q(z) \triangleq p(x>z)=\int_{z}^{\infty} \frac{1}{\sqrt{2 \pi}} e^{-y^{2} / 2} d y .
$$

$P_{\min }$ defines the least delivered power, $P_{\mathrm{t}}$ denotes the transmitted power, $k$ indicates a nondimensional parameter that is related to the features of antenna and the mean attenuation of channel, $\gamma$ denotes the path loss exponent, $d$ describes the distance among the controller and the corresponding data-plane nodes, and $d_{0}$ denotes the reference distance for the antenna far field [15]. Their corresponding values could be calculated to approximate either an experimental or analytical model. $\psi_{d B}$ describes a Gaussiandistributed stochastic variable with zero mean and variance $\sigma_{\psi_{d B}}$. 
Within the situation where the messages transmitted via the source, including data-plane nodes or controller, was not effectively sent to the receiver, the quantity of retransmission is indicated by $n_{s c}$, where its distribution is described as

$$
P_{r}\left\{n_{s c}=i\right\}=p_{s c}\left(1-p_{s c}\right)^{i-1}, \quad i \in\{1,2,3, \cdots\}
$$

The nodes controlled by $c_{i}$ are indicated by $s_{i, c_{i}}$. Now, the rate of data transfer of the wireless link $r\left(c_{i}, s_{i, c_{i}}\right)$ could be obtained as

$$
r_{i}\left(c_{i}, s_{i, c_{i}}\right)=B \log _{2}\left(1+\frac{p_{t}\left(c_{i}\right) h\left(c_{i}, s_{i, c_{i}}\right)}{\sigma^{2}+I\left(c_{i}, s_{i, c_{i}}\right)}\right) .
$$

$\eta_{i}$ describes the communication traffic used by upgrading the flow tables. $h\left(c_{i}, s_{i, c_{i}}\right)$ indicates the channel gain among controller $c_{i}$ and node $s_{i, c_{i}}$ given via $c_{i}$, where it can be computed as $h=d^{-\gamma}$. B describes the bandwidth of the channel, and $\sigma^{2}$ indicates the white Gaussian noise variance.

The transfer delay among data-plane nodes and controller is given by

$$
t_{s c}=\frac{\eta_{i}}{r_{i}\left(c_{i}, s_{i, c_{i}}\right)} \text {. }
$$

Now, the expected transfer delay can be obtained as $2 t_{s c}$ $/ p_{s c}$.

According to the above analysis, the overall response delay can be obtained as

$$
D(y)=\sum_{s \in S} \sum_{c \in N_{c}} y_{s c}\left(\frac{2 t_{s c}}{p_{s c}}\right)
$$

As for EC, we mainly assume the energy used by the data exchange among data-plane nodes and controllers and the synchronization among controllers. The mentioned two kinds of EC are influenced by the controller deployment, illustrated individually.

The communication overhead of the assignment of node $s$ to controller $c$ is indicated through $w_{s c}^{\alpha}$. Assignment overhead is described as

$$
W_{\alpha}(y)=\sum_{s \in S} \sum_{c \in N_{c}} y_{s c} w_{s c}^{\alpha}
$$

Increasing the data-plane nodes managed by the controller increases the number of messages exchanged with other controllers. The overhead produced through the fixed rate messages exchange among controller $m$ and $n$ is indicated by $w_{m n}^{\text {con }}$, while the extra cost related to the controller $m$ 's load is denoted by $w_{m n}^{\text {add }}$.

In a multicontroller network, controllers communicate data with each other using a consensus protocol for synchronization. Based on [16], various consensus approaches may be employed by several controllers to attain synchronization goals. According to the presented structure, the leader-based case is discussed here.

In the leader-based approach, controllers just exchange data with the leader. The leader-based overhead can be described as

$$
W_{\mathrm{lb}}(x, y)=\sum_{m \in N_{c}} \sum_{n \in N_{c}} x_{m}\left(w_{m n}^{\mathrm{lb} \_ \text {con }}+w_{m n}^{\mathrm{lb} \_ \text {add }} \sum_{s \in S} y_{s m}\right)
$$

According to the mentioned analysis, the overall overhead can be obtained as

$$
W(x, y)=W_{\alpha}(y)+W_{\mathrm{lb}}(x, y)
$$

Considering the energy utilized through the abovementioned transition overhead, according to [17], the following EC model can be obtained, which describes the EC of the transmission of $k$ bit/s information:

$$
E_{\mathrm{t}}(k, d)= \begin{cases}k\left(E_{\mathrm{elec}}+\varepsilon_{\mathrm{fs}} d^{2}\right), & d<d_{\mathrm{max}}, \\ k\left(E_{\mathrm{elec}}+\varepsilon_{\mathrm{amp}} d^{4}\right), & d \geq d_{\mathrm{max}},\end{cases}
$$

where $E_{\text {elec }}=50 \mathrm{~nJ} / \mathrm{bit}, \varepsilon_{\mathrm{fs}}=10 \mathrm{pJ} / \mathrm{bit} / \mathrm{m}^{2}$, and $\varepsilon_{\mathrm{amp}}=0.0013$ $\mathrm{pJ} / \mathrm{bit} / \mathrm{m}^{4}$ describe the energy parameters of the amplifier of power with various distances. The maximum communication radius is obtained as $d_{\max }=\sqrt{\varepsilon_{\mathrm{fs}} / \varepsilon_{\mathrm{amp}}}$. The whole EC for transition and synchronization is given by

$$
E(x, y)=W(x, y)+\left(E_{\mathrm{elec}}+\varepsilon_{\mathrm{fs}} d^{2}\right)
$$

It is evident that the outspread deployment of more controllers within the network decreases the reaction delay because nodes can be managed at a lower distance. Nevertheless, the dense deployment of a smaller number of controllers decreases the EC for multicontroller synchronization. The mentioned two assessments are inconsistent and cannot be diminished at a similar time. Thus, an appropriate deployment approach should be obtained to make a balance between delay and EC and obtain the minimum attainable response delay and consumption. The weight parameter $\alpha$ $>0$ is chosen to balance between the mentioned two assessments and optimizing the controller deployment and assignment through solving the given minimization problem.

$$
\begin{gathered}
\min _{x, y} \alpha D(y)+E(x, y) \\
\text { subject to }(1-5) .
\end{gathered}
$$

The standard linearization can be utilized to convert the problem (17) to a Mix-Integer Programming (MIP) problem. Now, a mathematical optimizer like CPLEX can be adopted for its solution [18]. The results can be obtained using Algorithm 1. It is performed on the global controller for a constant time range. 


\section{Numerical Simulations}

In the current section, the presented approach's efficiency is evaluated through the mobile ad hoc networking interoperability and cooperation (MANIAC) datasets [19]. The ONOS is assumed to serve as the studied controller because it provides the deployment of a multicontroller. Mininet-WiFi is employed to perform simulations on similar topology in MANIAC to evaluate whether the presented approach can satisfy the desired goals. Mininet-WiFi incorporates novel categories to provide the possible addition of the mentioned wireless mobile devices in a Mininet network framework [20]. Moreover, measurements can be obtained through an actual traffic overhead and delay in a simulated approach [21].

The model parameters are chosen as an out-of-door ambient combining with a path loss and shadow channel for (6), $k=-31.54 \mathrm{dBm}, P_{\mathrm{t}}=24 \mathrm{dBm}, P_{\min }=-115 \mathrm{dBm}, \gamma=$ 3.7, $\sigma_{\psi_{A B}}=3.65 \mathrm{dBm}$, and $d_{0}=50 \mathrm{~m} \mathrm{[15].} \mathrm{And} B=5 \mathrm{MHz}$ and $\sigma^{2}=-100 \mathrm{dBm}$ for (9). The packet scale $\eta$ of the requested flow tables is chosen as 1500 bytes. The traffic of controller nodes and synchronization of controllercontroller are utilized to generate the overhead. A certain amount is related to the controllers' type and their corresponding distance. The mathematical relations corresponding to the obtained overhead are utilized to obtain the EC. The CPLEX optimizer is adopted to solve the optimization problem.

Various controller deployment approaches are constructed based on the $k$-median clustering technique for optimizing the delay among data-plane nodes and controllers. The presented approach is compared with the $k$-median approach. As $k$-median considers the quantity of controllers as an initial data, a similar quantity of controllers is chosen for the comparison.

As shown in Figure 2, the presented approach can set the quantity of controllers corresponding to the parameter of weight $\alpha$. The weight parameter $\alpha$ denotes the same as that in (17). Increasing the weights of delay leads to more controllers in the vicinity of nodes. According to Figure 3, there is a trade-off between delay and EC. As shown in Figure 4, lower EC can be obtained through the presented approach than the $k$-median clustering approach. The MIP in this figure represents our presented approach since it has been converted to a MIP problem.

When the EC weights increase, the presented approach prefers to place the smaller number of controllers to reduce the intercontroller and controller-node communications and EC. When the response delay is more significant, the number of controllers positioned near the nodes to attain a controller with a lesser reaction delay is more. According to simulation outcomes, the presented approach can make a balance between delay and EC and regulate the proper quantity of controllers.

\section{Conclusions}

In the current work, the controller deployment issue is verified in the SDN-based TANET. A structure of SDN-based mobile ad hoc networks is constructed. Now, an optimal controllers' deployment is developed for delay guarantee and optimal EC. Simulation results indicate significant efficiency in decreasing delay and EC. According to the presented approach, there is a trade-off between two assessments in various preferences. The hybrid control in the mentioned framework and controller deployment can be considered as future works.

\section{Data Availability}

Previously reported CRAWDAD dataset was used to support this study and are available at https://crawdad.org/vt/ maniac/20110721/2009. These prior studies (and datasets) are cited at relevant places within the text as references [19].

\section{Conflicts of Interest}

The authors declare that they have no conflicts of interest.

\section{Acknowledgments}

This work is supported by the National Natural Science Foundation of China under Grant No. 61771354.

\section{References}

[1] K. Poularakis, G. Iosifidis, and L. Tassiulas, "SDN-enabled tactical ad hoc networks: extending programmable control to the edge," IEEE Communications Magazine, vol. 56, no. 7, pp. 132-138, 2018.

[2] R. Amin, M. Reisslein, and N. Shah, "Hybrid SDN networks: a survey of existing approaches," IEEE Communications Surveys \& Tutorials, vol. 20, no. 4, pp. 3259-3306, 2018.

[3] A. Giorgetti, A. Sgambelluri, R. Casellas, R. Morro, A. Campanella, and P. Castoldi, "Control of open and disaggregated transport networks using the open network operating system (ONOS) [invited]," IEEE/OSA Journal of Optical Communications and Networking, vol. 12, no. 2, pp. A171-A181, 2020.

[4] H. Pan and S. Wang, "Optimizing the SDN control-plane performance of the Openvswitch software switch," in 2015 IEEE Symposium on Computers and Communication (ISCC), pp. 403-408, Larnaca, Cyprus, 2015.

[5] H. C. Yu, G. Quer, and R. R. Rao, "Wireless SDN mobile ad hoc network: from theory to practice," in 2017 IEEE International Conference on Communications (ICC), pp. 1-7, Paris, 2017.

[6] P. Bellavista, A. Dolci, and C. Giannelli, "MANET-oriented SDN: motivations, challenges, and a solution prototype," in 2018 IEEE 19th International Symposium on "A World of Wireless, Mobile and Multimedia Networks" (WoWMoM), pp. 14-22, Chania, Greece, 2018.

[7] W. Qi, Q. Song, X. Kong, and L. Guo, “A traffic-differentiated routing algorithm in flying ad hoc sensor networks with SDN cluster controllers," Journal of the Franklin Institute, vol. 356, no. 2, pp. 766-790, 2019.

[8] K. Poularakis, Q. Qin, E. M. Nahum, M. Rio, and L. Tassiulas, "Flexible SDN control in tactical ad hoc networks," Ad Hoc Networks, vol. 85, pp. 71-80, 2019. 
[9] M. J. Abdel-Rahman, E. A. Mazied, K. Teague, A. B. MacKenzie, and S. F. Midkiff, "Robust controller deployment and assignment in software-defined cellular networks," in The 26th International Conference on Computer Communications and Networks (ICCCN 2017), pp. 1-9, Vancouver, BC, 2017.

[10] M. J. Abdel-Rahman, E. A. Mazied, A. MacKenzie, S. Midkiff, M. R. Rizk, and M. El-Nainay, "On stochastic controller placement in software-defined wireless networks," in 2017 IEEE Wireless Communications and Networking Conference (WCNC), pp. 1-6, San Francisco, CA, 2017.

[11] A. Dvir, Y. Haddad, and A. Zilberman, "Wireless controller deployment problem," in 15th IEEE Annual Consumer Communications \& Networking Conference (CCNC), pp. 1-4, Las Vegas, NV, 2018.

[12] M. Johnston and E. Modiano, "Controller placement in wireless networks with delayed CSI," IEEE/ACM Transactions on Networking, vol. 25, no. 3, pp. 1775-1788, 2017.

[13] M. Abolhasan, J. Lipman, W. Ni, and B. Hagelstein, "Softwaredefined wireless networking: centralized, distributed, or hybrid?," IEEE Network, vol. 29, no. 4, pp. 32-38, 2015.

[14] X. Wang, M. Huang, and L. Wan, "Joint 2D-DOD and 2DDOA estimation for coprime EMVS-MIMO radar," Circuits, Systems, and Signal Process, vol. 40, no. article 1605, 2021.

[15] A. Goldsmith, Wireless Communications, Cambridge University Press, New York, 2012.

[16] Q. Qin, K. Poularakis, G. Iosifidis, S. Kompella, and L. Tassiulas, "SDN controller placement with delay-overhead balancing in wireless edge networks," IEEE Transactions on Network and Service Management, vol. 15, no. 4, pp. 14461459, 2018.

[17] C. Li, J. Bai, J. Gu, X. Yan, and Y. Luo, "Clustering routing based on mixed integer programming for heterogeneous wireless sensor networks," Ad Hoc Networks, vol. 72, pp. 81-90, 2018.

[18] M. Barros and M. Casquilho, "Linear programming with CPLEX: an illustrative application over the Internet CPLEX in Fortran 90," in 2019 14th Iberian Conference on Information Systems and Technologies (CISTI), pp. 1-6, Coimbra, Portugal, 2019.

[19] L. A. DaSilva, A. B. MacKenzie, M. S. Thompson, and E. Q. Baumann, "The MANIAC challenge: educational experiences in ad hoc networking," IEEE Pervasive Computing, vol. 8, no. 1, pp. 7-11, 2009.

[20] R. R. Fontes, S. Afzal, S. H. B. Brito, M. A. S. Santos, and C. E. Rothenberg, "Mininet-WiFi: emulating software-defined wireless networks," in 2015 11th International Conference on Network and Service Management (CNSM), pp. 384-389, Barcelona, Spain, 2015.

[21] X. Wang, L. T. Yang, D. Meng, M. Dong, K. Ota, and H. Wang, "Multi-UAV cooperative localization for marine targets based on weighted subspace fitting in SAGIN environment," IEEE Internet of Things Journal, vol. 8, 2021. 